\title{
MODELO GRAVIMÉTRICO EN EL BORDE NORESTE DEL MACIZO NORPATAGÓNICO
}

\author{
Federico Lince Klinger ${ }^{1}$, Patricia Martinez ${ }^{1}$, Augusto E. Rapalini², Mario E. Gimenez ${ }^{1}$, \\ Mónica G. López De Luchi ${ }^{3}$, Flavia A. Croce ${ }^{1}$ y Francisco Ruiz ${ }^{1}$ \\ Recebido em 25 fevereiro, 2009 / Aceito em 17 setembro, 2009 \\ Received on February 25, 2009 / Accepted on September 17, 2009
}

\begin{abstract}
We present a gravimetric study on the northeast edge of the North Patagonian massif in the province of Rio Negro, to ascertain the depth distribution of the different outcrop units in the study area, as well as the geometry of the Navarrete Plutonic Complex. The negative anomalies in the Bouguer residual map reproduce the lateral density contrast between what would be the bedrock of Cambrian age and the lithologic units of the Mesozoic age. The solutions of the analytical signal along with variations in the curve of the horizontal gradient of gravity, obtained along a profile across the outcrops of the Navarrete Plutonic Complex, show contacts between the intrusive body and the bedrock with different densities. Over this profile we obtained a 2D (dimensional) gravimetric forward model adjusted with the density data of the surface rocks. Above the outcrop of the Yaminué Complex there exists a gravimetric high which does not correspond to the density in this area. This is a new evidence that proves that it is a series of intrusions and thin subhorizontal tectonic slices and that its thickness is probably thin. On the other hand the Navarrete Pluton shows the greatest development in size and depth to the east of Treneta stream.
\end{abstract}

Keywords: gravimetry, Navarrete Pluton, through, Yaminué Complex.

RESUMEN. Se presenta un estudio gravimétrico en el borde noreste del macizo Norpatagónico provincia de Río Negro, con el objeto de conocer la distribución en profundidad de las distintas unidades aflorantes en el área de estudio, como así también, la geometría del Complejo Plutónico Navarrete. Las anomalías negativas en el mapa residual de Bouguer reproducen el cambio lateral de densidad entre lo que sería el basamento de edad Cámbrica y las unidades litológicas de edad Mesozoica. Las soluciones de la señal analítica junto a las variaciones en la curva del gradiente horizontal de la gravedad, obtenidas a lo largo de un perfil transversal a los afloramientos del Complejo Plutónico Navarrete, evidencian contactos entre el cuerpo intrusivo y la roca de caja de diferente densidad. Sobre este perfil se obtiene un modelo de inversión gravimétrico 2D ajustado con datos de densidad de rocas en superficie. Sobre el afloramiento del Complejo Yaminué existe un máximo gravimétrico que no se corresponde con su densidad en superficie. Esto es una nueva evidencia de que el mismo es una serie de intrusiones y escamas tectónicas delgadas subhorizontales y que su espesor es probablemente de poca magnitud. Por otro lado el Plutón Navarrete muestra mayor desarrollo en su tamaño y profundidad hacia el este del arroyo Treneta.

Palabras-clave: gravimetría, plutón Navarrete, profundidades, Complejo Yaminué.

\footnotetext{
${ }^{1}$ CONICET, Instituto Geofísico Sismológico Volponi (IGSV), Facultad de Ciencias Exactas, Físicas y Naturales, Universidad Nacional de San Juan, Ruta 12, km 17 (Jardín de los Poetas) C.P. 5407, Marquesado, San Juan, Argentina. E-mails: flklinger@hotmail.com; patricia.signos@gmail.com; mgimenez@UNSJ-cuim.edu.ar; flacroce@gmail.com; drfranciscoruiz@gmail.com

${ }^{2}$ CONICET, Instituto Geofísico Daniel Valencio (INGEODAV), Facultad de Ciencias Exactas y Naturales, Universidad de Buenos Aires Pab.2, Ciudad Universitaria, C1428EHA, Bs.As., Argentina - E-mail: rapalini@gl.fcen.uba.ar

${ }^{3}$ CONICET, Instituto de Geocronología y Geología Isotópica (INGEIS), Universidad de Buenos Aires, Pabellon (INGEIS), Ciudad Universitaria, C1428EHA, Bs.As., Argentina - E-mail: deluchi@ingeis.uba.ar
} 


\section{INTRODUCCIÓN}

La evolución pre-mesozoica del borde NE del macizo Norpatagónico (MNP) ha sido objeto de numerosos estudios obteniéndose diversos resultados (ver ubicación en Fig. 1). Ramos (1984, 2008) propone que es un margen acrecional producto de la colisión entre la Patagonia y el borde SW de Gondwana en el Paleozoico tardío. Por otro lado, Dalla Salda et al. (1990) señalan la prolongación en el MNP de varias unidades geológicas paleozoicas aflorantes en las Sierras Pampeanas y proponen entonces un origen autóctono para el MNP.

Los principales rasgos geológicos del sector NE del MNP consisten en un basamento metamórfico de bajo grado de probable edad cámbrica intruído por plutonitas ordovícicas no deformadas a las que se superponen depósitos epiclásticos plegados de edad siluro-devónica (Rapalini et al., 2007). Un importante magmatismo, expresado como granitoides con diversos grados de deformación, afectó el NE del MNP en el Paleozoico tardío y Mesozoico temprano (Rapalini et al., 2008).

Las rocas ígneas gondwánicas aflorantes en esta región componen dos asociaciones principales, el Complejo Plutónico Navarrete y el Complejo Plutónico-Volcánico Treneta (Caminos, 1983; Llambías \& Rapela, 1984; Pankhurst et al., 1993). Estos se encuentran emplazados en la Formación Nahuel Niyeu (Pankhurst et al., 2006) y en el Complejo Plutónico Yaminué (Caminos, 1983; Basei et al., 2002) asignado este último, con dudas, al Carbonífero Tardío. La Formación Nahuel Niyeu es una secuencia metasedimentaria compuesta por metagrauvacas, lutitas pizarreñas y filitas. El Complejo Yaminué está formado principalmente por gneises y granitoides foliados.

Entre las localidades de Valcheta y Nahuel Niyeu se encuentran las sedimentitas marinas de la Formación Sierra Grande de edad Silúrica Tardía - Devónica Inferior, cubiertas en gran parte por el cuaternario (Caminos, 2001).

Las volcanitas Treneta, de probable edad Triásica descansan sobre la granodiorita Navarrete en un plano de discordancia erosiva. El Granito Flores, la más joven de las unidades plutónicas, está integrado por un conjunto de cuerpos intrusivos de dimensiones moderadas a pequeñas. Sus principales componentes son granitos masivos, de color rosado pálido, pobres en minerales máficos. Su edad es Jurásico Inferior. Durante el Cenozoico se produjeron en el macizo Norpatagónico varios episodios basálticos tipo plateau, entre los que se encuentran los basaltos olivínicos de la Formación Somún Curá (Ardolino, 1981). Su emplazamiento fue atribuido a procesos de inestabilidad térmica (Kay et al., 1992). Aunque las edades disponibles abarcan un amplio rango temporal, las efusiones se desarrollaron principal- mente en el Oligoceno, a partir de los $33 \mathrm{Ma}$ (Ardolino \& Franchi, 1993).

Con objeto de aportar evidencias que ayuden a esclarecer la historia evolutiva del MNP, en alguno de los sentidos descriptos anteriormente, se realizó un trabajo multidisciplinario que involucró estudios: petrológico, paleomagnético, geobarométrico, magnetométrico y gravimétrico (Rapalini et al., 2010). En este trabajo se presentan en detalle los resultados de un estudio gravimétrico que consistió en realizar un modelo gravimétrico 2D con objeto de determinar los espesores de las distintas unidades aflorantes en el área de interés.

\section{METODOLOGÍA}

Se relevaron 144 puntos gravimétricos y topográficos en la región NE del MNP, partiendo de la localidad de Valcheta hacia Nahuel Niyeu, Corral Chico, Rincón de Treneta, y Chipauquil. Los valores de gravedad se obtuvieron con un gravímetro LaCoste \& Romberg, con precisión $\pm 0,01 \mathrm{mGal}$. Las mediciones gravimétricas fueron referidas al IGSN71 (International Gravity Standardization Net 1971) (Morelli et al., 1974) y vinculadas a la estación fundamental de Miguelete que se localiza en la provincia de Buenos Aires, Argentina.

En cada estación gravimétrica se determinó la altura elipsoidal referida al WGS84 (World Geodetic System 1984), utilizando dos GPS de simple frecuencia y precisión submétrica. En el campo se dispuso de un equipo GPS base y otro móvil con un radio máximo de $20 \mathrm{~km}$ de separación entre ellos. Ambos equipos registraron en modo diferencial.

Se realizaron mediciones de densidad, en muestras de mano, de las litologías aflorantes más representativas del área de estudio (Tab. 1). Se empleó el método de la doble pesada recubriendo cada muestra con parafina, el error cometido en la determinación fue del orden de la milésima de $\mathrm{g} / \mathrm{cm}^{3}$. Cada medición presentada en dicha tabla consistió en el promedio de 6 a 10 muestras individuales aflorantes en el mismo sitio. Estos sitios están ampliamente distribuidos en el área de interés (Fig. 1).

Estos valores de densidad obtenidos indican que la Formación Nahuel Niyeu, caja de los complejos Navarrete y Yaminué (Llambías et al., 2002; Rapalini et al., 2010) es la unidad de mayor densidad aflorante $\left(2,72 \mathrm{~g} / \mathrm{cm}^{3}\right)$. Tanto el Complejo Yaminué como el Navarrete poseen densidades medias semejantes de 2,64 y 2,63 g/ $\mathrm{cm}^{3}$ respectivamente. Sin embargo puede observarse una dispersión mucho mayor de valores en el primero de los complejos consistente con su mayor variación litológica. El Granito Flores posee una densidad significativamente menor $\left(2,59 \mathrm{~g} / \mathrm{cm}^{3}\right)$ coherente con su composición más ácida. 


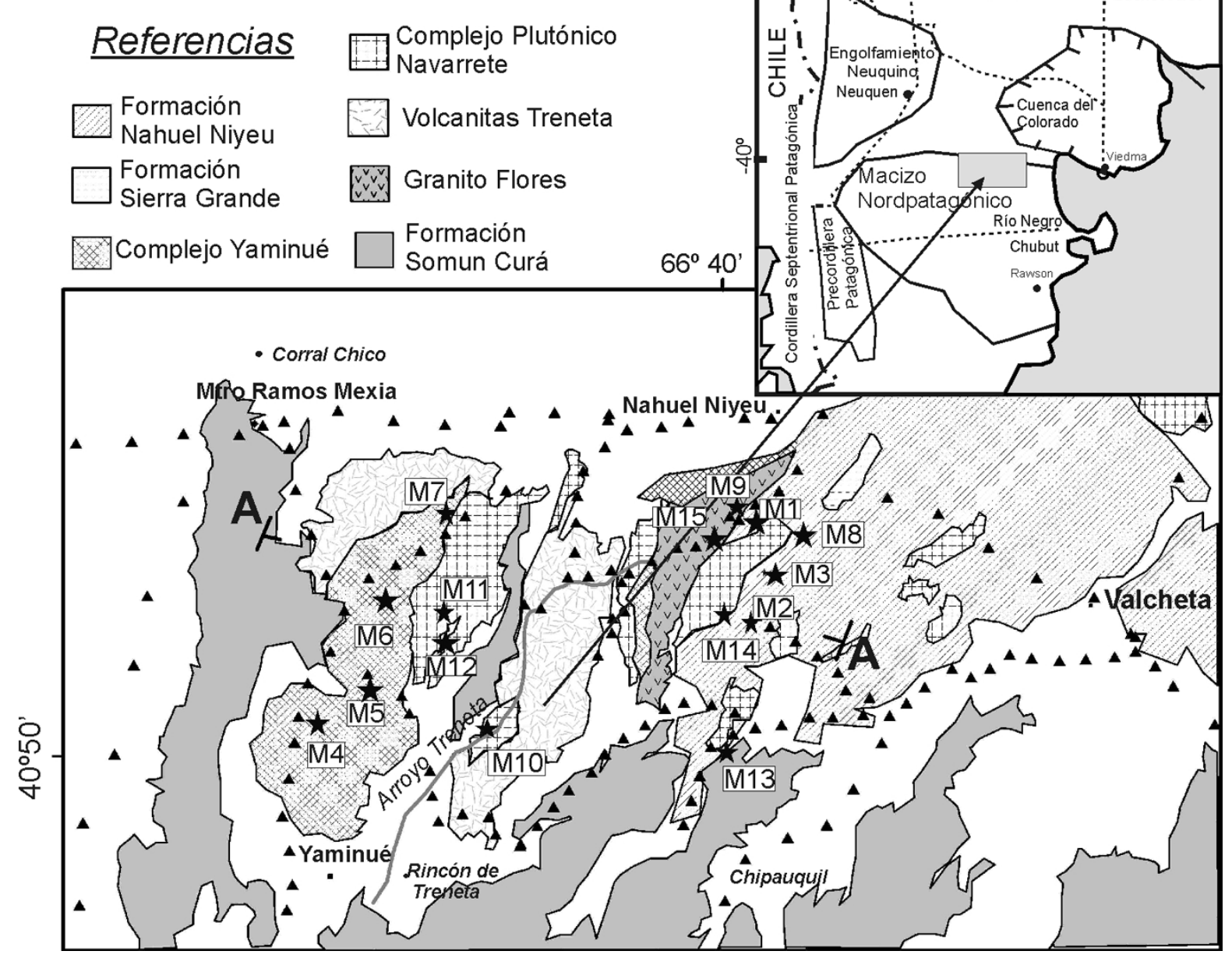

Figura 1 - Mapa geológico del área de estudio, en este se han volcado las formaciones geológicas de mayor relevancia. (ム) Estaciones gravimétricas, ( $\star$ ) Determinación de densidad.

\section{Procesamiento de datos gravimétricos}

Las variaciones laterales de densidad existentes en la corteza pueden ser individualizadas a través de las anomalías gravimétricas (Blakely, 1995). La anomalía de Bouguer completa se calcula como la diferencia de gravedad entre el valor teórico y el observado reducido al nivel medio del mar a través de las clásicas correcciones de aire libre $(0,3086 \mathrm{~h})$, Bouguer $(0,1118 \mathrm{~h})$ con densidad para una losa plana infinita de $2,67 \mathrm{~g} / \mathrm{cm}^{3}$ y topográfica. Para la corrección topográfica se utilizaron dos modelos de elevación digital, uno regional y otro local, obtenidos del Shuttle Radar Topography Mission (SRTM) del Servicio Geológico de Estados Unidos (USGS). El programa utilizado combina los algoritmos desarrollados por Kane (1962) y por Nagy (1966) obteniendo una grilla de corrección topográfica la cual, a través de una operación de muestreo, le asigna el valor de la corrección a cada estación gravimétrica. Los valores de anomalía fueron regularizados con una distancia de $3 \mathrm{~km}$ de separación aplicando el método de grillado de Mínima Curvatura (Briggs, 1974). Esta metodología es la aconsejada pues regulariza datos geofísicos creando una grilla de valores estimados a partir de datos distribuidos en forma no uniforme sobre el terreno. Con los valores grillados se preparó el mapa de anomalía de Bouguer completa correspondiente a la zona de estudio (Fig. 2).

\section{Filtrado de anomalías}

El mapa de anomalía de Bouguer, (Fig. 2), contiene distintos efectos gravimétricos producidos por estructuras de carácter regional más el efecto gravimétrico debido a estructuras geológicas de carácter local comprendidas en la corteza superior.

Para relacionar las anomalías gravimétricas con el complejo 
gondwánico emplazado en corteza superior, es necesario descontar del mapa de anomalía de Bouguer completa el efecto gravimétrico producido por las estructuras regionales.

Tabla 1 - Tabla de densidades según el muestreo visto en la Figura 1. Describe la unidad geológica asignada a cada sitio: M1 hasta M15, N representa la cantidad de muestras en cada sitio. La densidad promedio para cada sitio y la densidad promedio para cada unidad geológica.

\begin{tabular}{|c|c|c|c|c|}
\hline Unidad Geológica & Sitio & $\mathrm{N}$ & $\begin{array}{c}\text { Densidad } \\
\left(\mathrm{g} / \mathrm{cm}^{3}\right)\end{array}$ & $\begin{array}{c}\text { Densidad } \\
\text { Promedio } \\
\left(\mathrm{g} / \mathrm{cm}^{3}\right)\end{array}$ \\
\hline \multirow{3}{*}{ F. Nahuel Niyeu } & M1 & 6 & 2,747 & \multirow{3}{*}{$2,72 \pm 0,03$} \\
\hline & M2 & 7 & 2,691 & \\
\hline & M3 & 6 & 2,714 & \\
\hline \multirow{4}{*}{ Complejo Yaminué } & M4 & 8 & 2,725 & \multirow{4}{*}{$2,64 \pm 0,06$} \\
\hline & M5 & 9 & 2,637 & \\
\hline & M6 & 10 & 2,611 & \\
\hline & M7 & 8 & 2,599 & \\
\hline \multirow{6}{*}{$\begin{array}{c}\text { Complejo Plutónico } \\
\text { Navarrete }\end{array}$} & M8 & 7 & 2,625 & \multirow{6}{*}{$2,63 \pm 0,02$} \\
\hline & M10 & 8 & 2,660 & \\
\hline & M11 & 7 & 2,614 & \\
\hline & M12 & 7 & 2,649 & \\
\hline & M13 & 7 & 2,655 & \\
\hline & M14 & 7 & 2,654 & \\
\hline \multirow{2}{*}{ Granito Flores } & M9 & 7 & 2,603 & \multirow{2}{*}{$2,59 \pm 0,01$} \\
\hline & M15 & 8 & 2,584 & \\
\hline
\end{tabular}

Una forma de obtener el efecto gravimétrico regional es proIongar el campo potencial a una altura determinada sobre la superficie de medición (Pacino \& Introcaso, 1987). El método empleado se basa en la ecuación de Dirichlet (Dean, 1958). En el campo potencial prolongado se atenúan las anomalías de corta longitud de onda suavizando el efecto gravimétrico de cuerpos más superficiales, de forma tal, que sólo queda el efecto regional del campo gravitatorio. Siguiendo este método de separación de anomalías gravimétricas, se hicieron prolongaciones analíticas del campo potencial a diferentes alturas desde $10 \mathrm{a}$ $45 \mathrm{~km}$ con intervalos de $5 \mathrm{~km}$ obteniendo mapas de anomalía de Bouguer regionales. Del análisis cualitativo de estos mapas se opto por el mapa que resultó de prolongar el campo potencial a $35 \mathrm{~km}$ de altura (Fig. 3), debido a que es el que mejor representa las largas longitudes de onda que estamos buscando. En la Figura 3 se observa que las isoanómalas crecen negativamente hacia el macizo Norpatagónico, la Precordillera Patagónica y la Cordillera Septentrional Patagónica. Estos máximos negativos reflejan la fuerte componente horizontal del efecto gravimétrico de la raíz andina. Hacia el este las isoanómalas aumentan alcanzando el valor de $0 \mathrm{mGal}$ en la cuenca del Colorado. Este aumento es coherente con el efecto positivo que produce una disminución del espesor cortical continental hacia la costa Atlántica.

El efecto regional (Fig. 3) se descontó del mapa de anomalía de Bouguer completa (Fig. 2), obteniendo la anomalía residual de Bouguer (Fig. 4).

El mapa de anomalía residual de Bouguer manifiesta un máximo positivo de $15 \mathrm{mGal}$ hacia el extremo oeste donde los gneises y granitoides, de edad Carbonífera del Complejo Yaminué se encuentran cubiertos por las coladas basálticas de la Formación Somún Curá. Los valores positivos de la anomalía residual de Bouguer sobre la parte occidental del mapa, no se correlacionan con las densidades halladas en muestras de mano de la Formación Yaminué (2,64 g/ $\left.\mathrm{cm}^{3}\right)$ (ver Tab. 1). Por otro lado, sobre el borde este se registran otros positivos gravimétricos coincidentes con los afloramientos de la Formación Nahuel Niyeu. Estos valores positivos reflejan en buena medida la densidad $\left(2,72 \mathrm{~g} / \mathrm{cm}^{3}\right)$ de las rocas metamórficas de edad Cámbrica que constituirían el basamento del área.

Luego, las isoanómalas decrecen alcanzando máximos negativos hacia la parte central del mapa coincidiendo con el afloramiento del Complejo Navarrete, la porción norte del Granito Flores y afloramientos metamórficos del Complejo Yaminué. Las densidades obtenidas en superficie para estas unidades geológicas varían desde $2,63 \mathrm{~g} / \mathrm{cm}^{3}$ en los granitoides del Complejo Navarrete hasta 2,59 g/ $\mathrm{cm}^{3}$ en el Granito Flores. El mapa de isoanómalas residuales no discrimina entre estas últimas unidades geológicas. Su exposición asociada al mínimo gravimétrico central, indica que este rasgo geofísico estaría determinado por la presencia de niveles estructurales profundos. Niveles basamentales más someros se corresponderían con los máximos oriental y occidental. Coincidentemente, no se observan en ellos afloramientos de unidades epiclásticas no metamórficas pre-Mesozoicas.

\section{Señal analítica}

Con el objeto de determinar los bordes entre las diferentes unidades geológicas se aplicó a lo largo de un perfil A-A', Figura 5 (arriba), la técnica de señal analítica 2D siguiendo la metodología propuesta por Nabighian (1972). Este perfil es perpendicular a los afloramientos del área y los atraviesa alternativamente según lo observamos en la Figura 5 (arriba). Los saltos en la curva de gradiente horizontal, Figura 5 (centro, líneas de trazos), denotan cambios abruptos en la densidad de la roca que constituye la corteza más superficial. En el área de estudio la cobertura sedimentaria es de escaso espesor, lo que permite reconocer en superficie los contactos entre las diferentes estructuras aflorantes. 


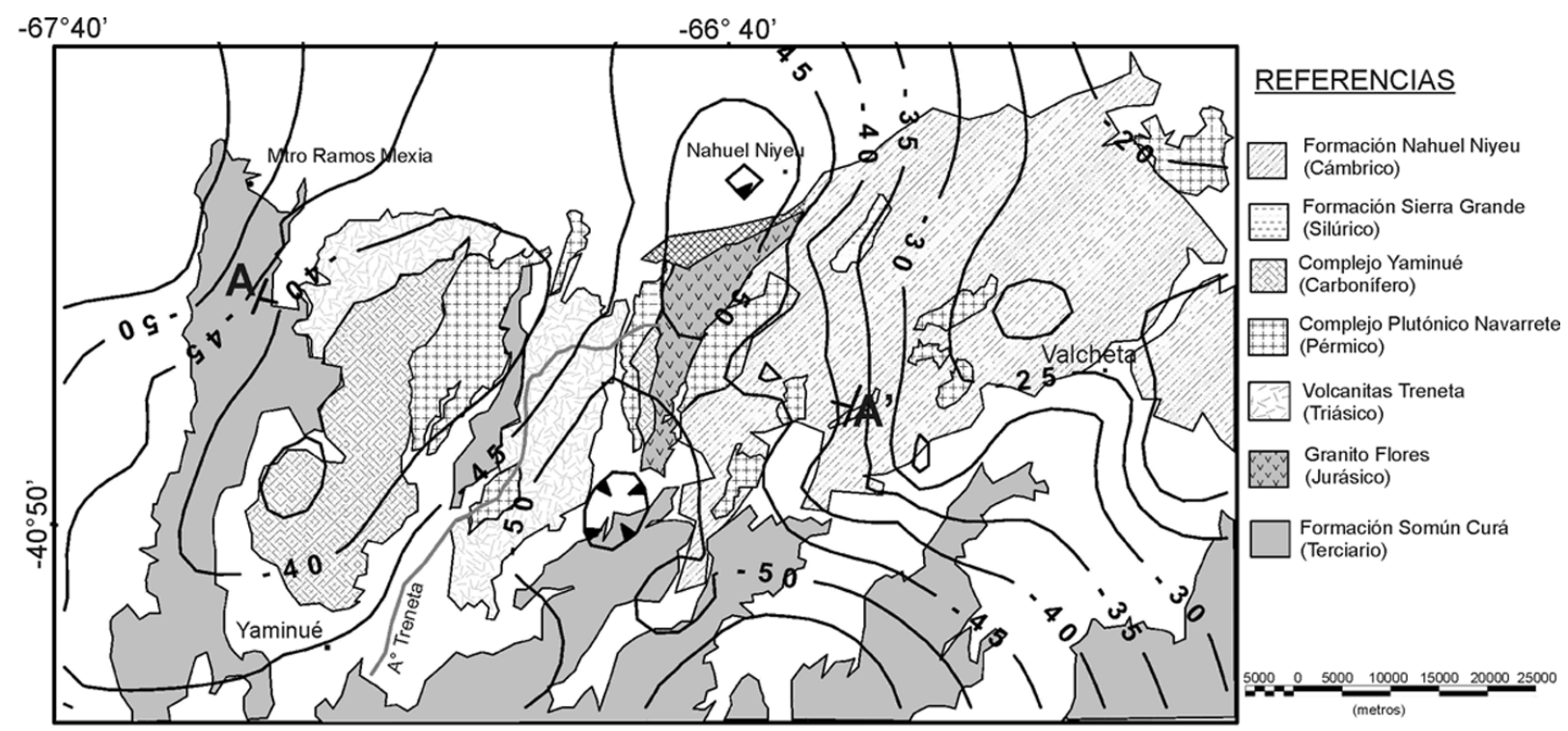

Figura 2 - Mapa de anomalía de Bouguer completa calculada en el Sistema Internacional de Referencia de 1971. Método de grillado Mínima Curvatura. Isoanómalas cada 5 mGal. A-A' perfil modelado en Figuras 5 y 6 .

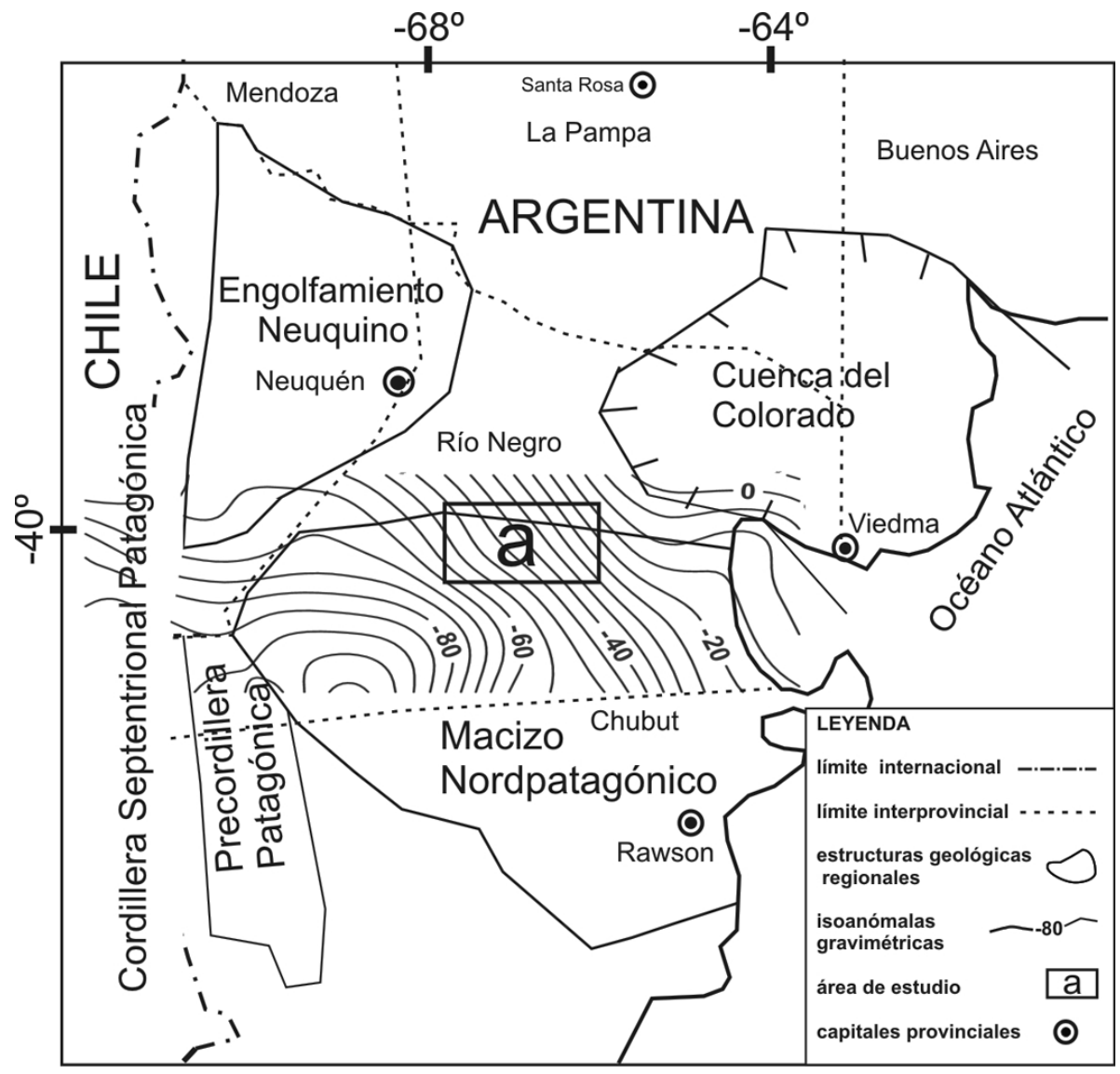

Figura 3 - Mapa de anomalías regionales de Bouguer. Isoanómalas cada 5 mGal obtenida por el método de prolongación ascendente del campo potencial a una altura de $35 \mathrm{~km}$. Las isoanómalas se extienden más allá del límite del área de estudio (a) con el propósito de eliminar el efecto de bordes. 
$-67^{\circ} 40^{\prime}$

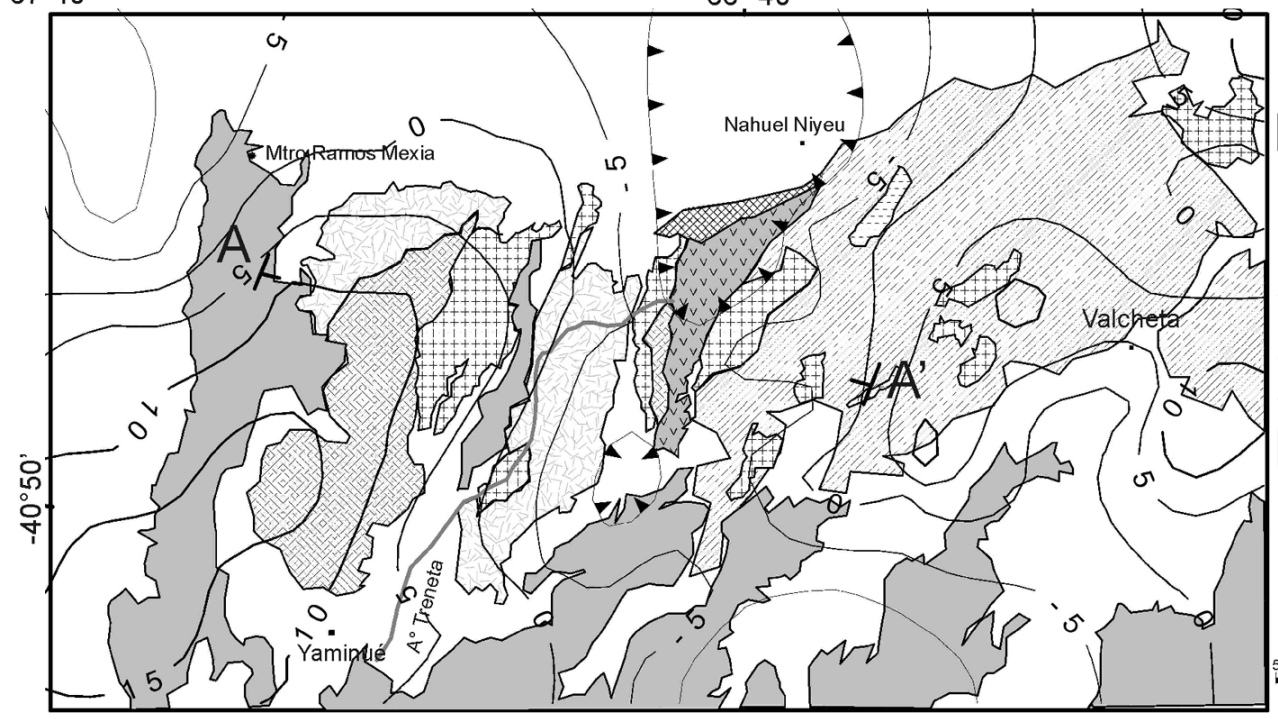

REFERENCIAS

Formación Nahuel Niyeu (Cámbrico)

Formación Sierra Grande (Silúrico)

Complejo Yaminue (Carbonífero)

Complejo Plutónico Navarrete (Pérmico)

Volcanitas Treneta (Triásico)

Granito Flores (Jurásico)

Formación Somún Curá (Terciario)

Figura 4 - Mapa de anomalías residuales de Bouguer sobre los afloramientos de las formaciones más importantes en la región. Isoanómalas cada 5 mGal.
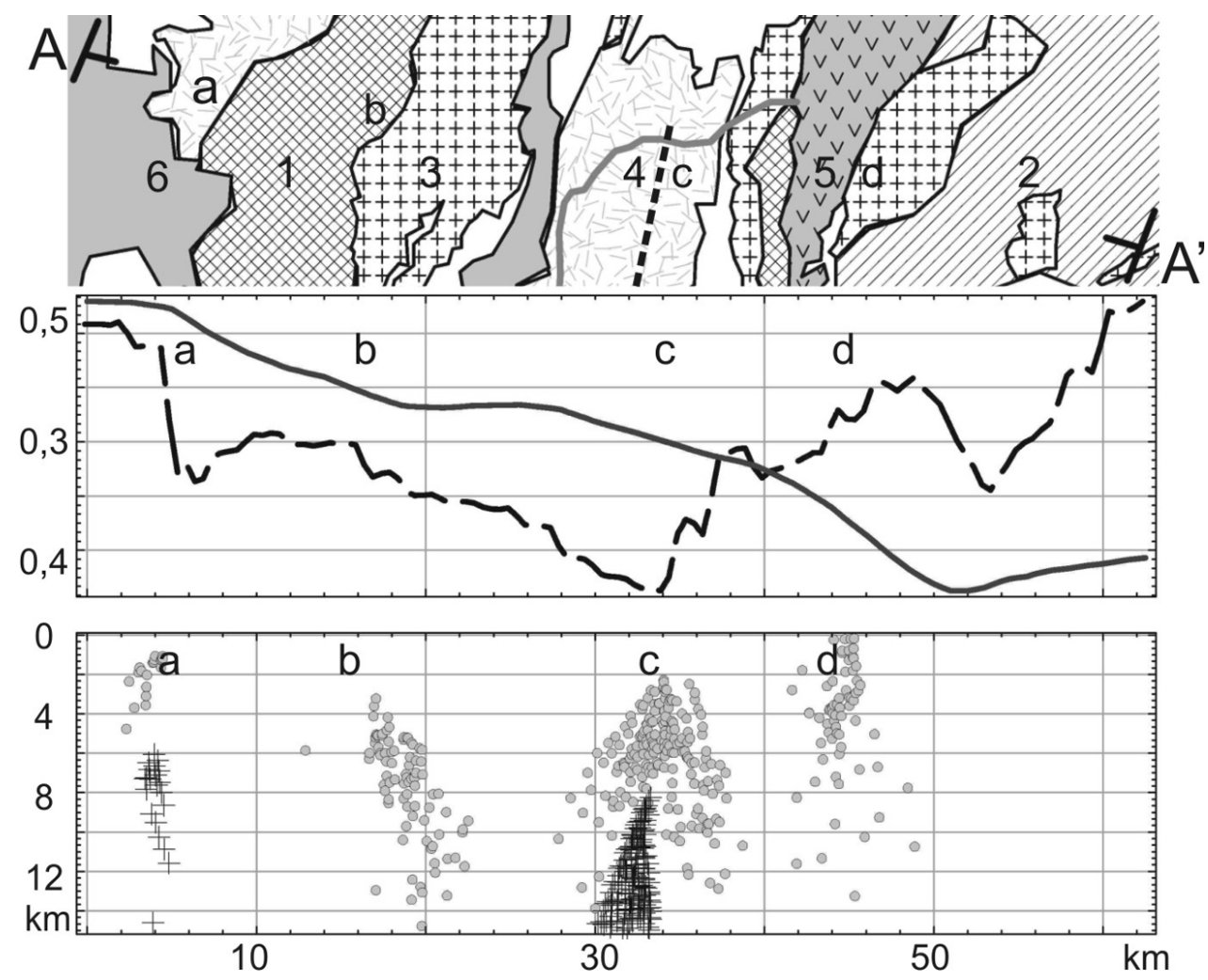

Figura 5 - Arriba: Perfil A-A' sobre mapa geológico las principales unidades geológicas se identifican con números del 1 al 6. 1) Complejo Yaminué; 2) Formación Nahuel Niyeu; 3) Complejo Plutónico Navarrete; 4) Formación Treneta; 5) Granito Flores; 6) Formación Somún Curá. Con línea de trazo negra se simboliza la falla interpretada a través de la geología de superficie. Centro: línea de trazo representa el gradiente horizontal y, la línea llena la altura sobre el nivel medio del mar. Abajo: Los círculos y las cruces simbolizan las soluciones de la señal analítica. Las letras a, b, c, d marcan alineaciones de soluciones asociadas a contrastes de densidad. 
Para obtener las soluciones de la señal analítica es necesario establecer el ancho de ventana móvil, y la profundidad de investigación. Para determinar el ancho de la ventana de búsqueda se consideraron las Iongitudes de onda de las anomalías más importantes del área. Igualmente, se probaron varias distancias para comprobar la sensibilidad del método encontrándose que a la distancia de $10 \mathrm{~km}$ los racimos de soluciones responden en buena forma a las estructuras de interés. Para distancias mayores las soluciones responden a estructuras más regionales y para distancias menores a $10 \mathrm{~km}$ se dificulta su interpretación.

Las alineaciones de las soluciones de la señal analítica muestran como se continúan en profundidad, los contactos entre cuerpos constituidos por rocas de diferente densidad.

Los racimos de soluciones de la señal analítica a lo largo del perfil A-A' son interpretados como (ver Fig. 5, abajo):

a) Contacto 0 falla que limita al Complejo Yaminué de una estructura de litología diferente. En superficie coincide con el contacto entre las coladas basálticas olivínicas de la Formación Somún Curá y el Complejo Yaminué.

b) Contacto o falla entre el Complejo Yaminué y el Complejo plutónico Navarrete, estas soluciones alcanzan $12 \mathrm{~km}$ de profundidad aproximadamente.

c) El cambio abrupto en la curva del gradiente horizontal coincide con las fallas interpretadas con datos de geología en superficie en el curso del arroyo Treneta. Se observan más de un racimo de soluciones contaminando la interpretación.

d) Contacto entre el Granito Flores y el Complejo plutónico Navarrete.

\section{Modelo gravimétrico}

La relación entre las anomalías gravimétricas y sus fuentes se cuantifica, en buena medida a través de modelos gravimétricos. Esta relación no es unívoca por lo que es necesario establecer límites iníciales que restrinjan el número de soluciones posibles. La escasa información geofísica en el área (líneas de sísmica de reflexión, pozos exploratorios) Ileva a aplicar técnicas de interpretación como la señal analítica (Nabighian, 1972) que sirven para localizar los bordes de las fuentes de anomalías gravimétricas y magnéticas. No obstante, en esta interpretación contamos con la ventaja de tener muestras con densidad, con lo cual se reduce el nivel de incertidumbre en el cálculo de la profundidad. Entonces a partir de la integración del resultado arrojado por el método de señal analítica y las densidades en muestras de mano, es posible determinar modelos gravimétricos coherentes con la información geológica de la región.

Con el fin de inferir la distribución de densidades en el subsuelo de la región de estudio, se ha modelado el perfil A-A' transversal a los afloramientos de interés. El modelo gravimétrico se ha realizado utilizando el programa GM-SYS que está basado en el método de Talwani et al. (1959).

Se calculó la respuesta gravimétrica a partir de información geológica Llambías et al. (2002) y Rapalini et al. (2010). Se construyó una sección geológica usando polígonos cerrados para representar cada unidad geológica. A cada polígono se le asignó un valor de densidad constante (Tab. 1). Luego la diferencia entre el dato observado y la respuesta calculada fue minimizada variando los vértices de los polígonos del modelo inicial.

\section{RESULTADOS Y CONCLUSIÓN}

En términos generales existe correlación entre el mapa de anomalía gravimétrica residual y la densidad de las distintas unidades aflorantes en el área de estudio excepto con las rocas expuestas del Complejo Yaminué. Los máximos gravimétricos oriental y occidental de mediana longitud de onda, coinciden con los asomos de la Formación Nahuel Niyeu y el Complejo Yaminué, este último con una densidad considerablemente menor a la de la Formación Nahuel Niyeu. Rapalini et al. $(2007,2010)$ han señalado que el Complejo Yaminué y los leucogranitos Cabeza de Vaca (considerados en la literatura previa como parte del Complejo Navarrete) registran evidencias de emplazamiento y deformación asociados a corrimientos de vergencia al SO. Von Gosen (2003) describió una deformación de similares características afectando la cubierta epiclástica de la Formación Sierra Grande y la Formación Nahuel Niyeu en el sector central del área de estudio. La estructura interna del Complejo Yaminué (Llambías et al., 2002; López de Luchi et al., 2008; Rapalini et al., 2010), su variación litológica, sus relaciones con la Formación Nahuel Niyeu y su asociación con un máximo gravimétrico que no se corresponde con su densidad sugieren que el mismo es probablemente una serie de intrusiones y escamas tectónicas delgadas subhorizontales y que su espesor es probablemente de poca magnitud.

El modelo gravimétrico a lo largo del perfil A-A' (Fig. 6) provee un medio para examinar lo anteriormente expresado relacionado con la prominente diferencia en la anomalía gravimétrica existente a ambos lados del arroyo Treneta. La causa de este fuerte gradiente parece estar relacionada con una falla localizada a lo largo de dicho arroyo. Esta estructura cortical divide en bloques el basamento constituido por rocas de la Formación Nahuel Niyeu. Hacia el oeste del perfil el espesor del basamento 

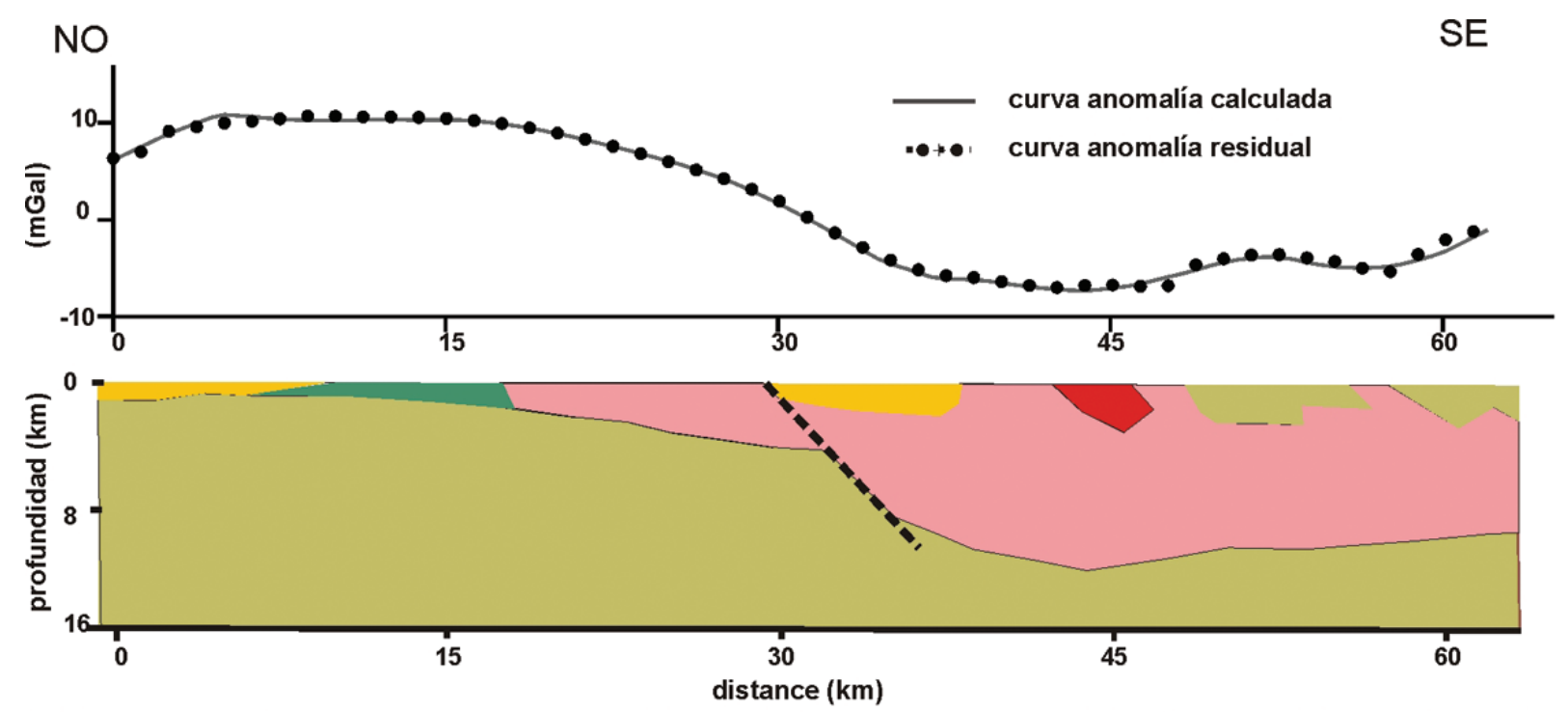

F. Nahuel Niyeu $\left(2,72 \mathrm{~g} / \mathrm{cm}^{3}\right) \square$ Complejo Yaminué $\left(2,64 \mathrm{~g} / \mathrm{cm}^{3}\right) \square$ Complejo Navarrete $\left(2,63 \mathrm{~g} / \mathrm{cm}^{3}\right) \square$ Granito Flores $\left(2,59 \mathrm{~g} / \mathrm{cm}^{3}\right)$

\section{Grupo Treneta $\left(2,61 \mathrm{~g} / \mathrm{cm}^{3}\right)$}

Figura 6 - Modelo de densidad a lo largo del perfil A-A' (ver ubicación en la Fig. 2). Las densidades utilizadas, fueron las obtenidas a través de muestreo (ver Tab. 1). Así para la Formación Nahuel Niyeu se utilizó: 2,72 g/ $\mathrm{cm}^{3}$; Complejo Navarrete, 2,63 g/ $/ \mathrm{cm}^{3}$; Complejo Yaminué, 2,64 g/ $/ \mathrm{cm}^{3}$; Granito Flores, 2,59 g/ $/ \mathrm{cm}^{3}$; Grupo Treneta, $2,61 \mathrm{~g} / \mathrm{cm}^{3}$.

aumenta considerablemente lo que justificaría el máximo gravimétrico sobre el afloramiento del Complejo Yaminué. Hacia la parte oriental del perfil el Complejo plutónico Navarrete (densidad 2,63 $\mathrm{g} / \mathrm{cm}^{3}$ ) aumenta en profundidad alcanzando los $12 \mathrm{~km}$ en coincidencia con el método radial espectral aplicado por Croce et al. (2009), para la misma zona de estudio.

La señal analítica sugiere la presencia de dos zonas de falla de vergencia opuesta limitando la zona de anomalía gravimétrica negativa central que permite interpretar el área como un bloque hundido, en la que se han preservado niveles corticales más someros. La presencia de volcanitas de la Formación Treneta (Triásico) expuestas en los distintos bloques del área sugiere que esta estructuración debería ser previa a la extrusión de las mismas.

\section{AGRADECIMIENTOS}

Los autores agradecen a la Agencia Nacional de Promoción Científica y Tecnológica - Argentina por los subsidios otorgados PICTR 00283 y PICT 2005-Redes-33630; también al CICITCAUNSJ - con sus proyectos No. E816, No. E815, y No. E810, y al Consejo Nacional de Promoción Científica y Tecnológica (CONICET) de Argentina, a través del Proyecto PIP 6044, que permitieron solventar las campañas de relevamiento de datos de campo.

\section{REFERENCIAS}

ARDOLINO AA. 1981. El vulcanismo cenozoico del borde suroriental de la meseta de Somún Curá. In: VIII Congr. Geol. Arg., Actas 3: 7-23. Provincia del Chubut. Argentina.

ARDOLINO AA \& FRANCHI MR. 1993. El vulcanismo cenozoico de la Meseta de Somún Curá, Provincias de Río Negro y Chubut. In: XII Congr. Geol. Arg. y II Congr. de Exploración de Hidrocarburos, Actas 4: 225235. Mendoza. Argentina.

BASEI MAS, VARELA R, SATO AM, SIGA JRO \& LLAMBÍAS EJ. 2002. Geocronología sobre rocas del Complejo Yaminué, Macizo Norpatagónico, Río Negro, Argentina. In: XV Congr. Geol. Arg., Actas 3: 117-122. El Calafate. Argentina.

BLAKELY R. 1995. Potential theory in gravity and magnetic applications. Cambridge University Press. 441 pp.

BRIGGS IC. 1974. Machine contouring using minimum curvature. Geophysics, 39: 39-48.

CAMINOS R. 1983. Descripción Geológica de las Hojas 39g, Cerro Tapiluke y 39h, Chipauquil, Provincia de Río Negro. Servicio Geológico Nacional, 41 pp (inédito).

CAMINOS R. 2001. Descripción Geológica de la Hoja 4166-I, Valcheta, Provincia de Río Negro. Buenos Aires, Servicio Geológico Minero Argentino. Boletín 310, 78 pp. 
CROCE F, LINCE KLINGER F, GIMENEZ M, MARTINEZ MP \& RUIZ F. 2009. Estimación de profundidades del Complejo plutónico Navarrete mediante procesamiento gravimétrico. Revista Geoacta, 34: 1-8.

DALLA SALDA L, CINGOLANI C \& VARELA R. 1990. The origin of Patagonia. Comunicaciones, Una revista de geología andina, 41: 55-61.

DEAN WC. 1958. Frequency analysis for gravity and magnetic interpretation. Geophysics, 23: 97-122.

KANE MF. 1962. A comprehensive system of terrain corrections using a digital computer. Geophysics, 27: 455-462.

KAY SM, ARDOLINO AA, FRANCHI MR \& RAMOS VA. 1992. The Somuncura plateau: An Oligo-Miocene 'baby-hotspot' in extra- Andean Patagonia (40.5 $5^{\circ}$ to $43^{\circ} \mathrm{S}$ latitude). EOS Transactions, American Geophysical Union 73, p. 337.

LÓPEZ DE LUCHI MG, WEMMER K \& RAPALINI AE. 2008. The cooling history of the North Patagonian Massif: first results for the granitoids of the Valcheta area, Río Negro, Argentina. In: LINARES E, CABALERI NG, DO CAMPO MD, DUCÓS EI \& PANARELLO HO (Eds.). Book of Abstracts. VI South American Symposium on Isotope Geology. San Carlos de Bariloche, Abstracts, p. 33.

LLAMBÍAS EJ \& RAPELA CW. 1984. Geología de los Complejos Eruptivos de La Esperanza, provincia de Río Negro. Revista de la Asociación Geológica Argentina, 39: 220-243.

LLAMBÍAS EJ, VARELA R, BASEI M \& SATO AM. 2002. Deformación y metamorfismo Neopaleozoico en Yaminué, Macizo Norpatagónico (4050'S, 6740'W): su relación con la Fase Orogénica San Rafael y el arco de los Gondwánides. In: XV Congr. Geol. Arg. Actas 3: 123-128. El Calafate. Argentina.

MORELLI C, GANTAR C, HONKASALON T, MCCONNEL K, TANNER JG, SZABO B, UOTILA U \& WHALEN CT. 1974. The International Gravity Standardization Net 1971 (IGSN71), IUGG-IAG Publ. Spec. 4, Int. Union of Geod. and Geophys., Paris. 194 pp.

NABIGHIAN MN. 1972. The analytic signal of two-dimensional magnetic bodies with polygonal cross-section: its properties and use for automated anomaly interpretation. Geophysics, 37: 780-786.

NAGY D. 1966. The Gravitational Attraction of a Right Rectangular Prism. Geophysics, 30: 362-371.
PACINO MC \& INTROCASO A. 1987. Regional anomaly determination using the upwards continuation method. Bollettino di Geofisica Teorica ed Applicata, 29: 113-122.

PANKHURST RJ, CAMINOS R \& RAPELA CW. 1993. Problemas Geocronológicos de los Granitoides Gondwánicos de Nahuel Niyeu, Macizo Norpatagónico. In: XII Congr. Geol. Arg. y II Congr. de Exploración de Hidrocarburos, Actas 4: 99-104. Mendoza. Argentina.

PANKHURST RJ, RAPELA CW, FANNING CM \& MÁRQUEZ M. 2006. Gondwanide continental collision and the origin of Patagonia. EarthScience Reviews, 76: 235-257.

RAMOS VA. 1984. Patagonia: ¿Un continente paleozoico a la deriva? In: IX Congr. Geol. Arg., Actas 2: 311-325. San Carlos de Bariloche. Argentina.

RAMOS VA. 2008. Patagonia: a Paleozoic continent adrift? Journal of South American Earth Sciences, 26(3): 235-251.

RAPALINI AE, LÓPEZ DE LUCHI MG, TOMEZZOLI R, LINCE KLINGER F, GIMENEZ ME \& MARTINEZ P. 2007. Magnetic fabric of the plutonic Navarrete Complex: further evidence for a Late Paleozoic collisional event in Northern Patagonia? In: GEOSUR, Abstracts. Santiago. Chile. CD-ROM.

RAPALINI AE, LÓPEZ DE LUCHI MG, CROCE F, LINCE KLINGER F, TOMEZZOLI R \& GIMENEZ ME. 2008. Estudio geofísico del Complejo Plutónico Navarrete: implicancias para la evolución tectónica de Patagonia en el Paleozoico Tardío. In: V Simposio Argentino del Paleozoico Superior, Museo Arg. Ciencias Naturales, Bs. As., Resúmenes, p. 34.

RAPALINI AE, LÓPEZ DE LUCHI MG, MARTINEZ DOPICO C, LINCE KLINGER F, GIMENEZ ME \& MARTINEZ MP. 2010. Did Patagonia collide against Gondwana in the Late Paleozoic? Some insights from a multidisciplinary study of magmatic units of the North Patagonian Massif. Geologica Acta, 8(3): En prensa.

TALWANI M, WORZEL JL \& LANDISMAN M. 1959. Rapid gravity computations for two-dimensional bodies with application to the Mendocino submarine fracture zone. Journal of Geophysical Research, 64: 49-58.

VON GOSEN W. 2003. Thrust tectonics in the North Patagonian Massif (Argentina): Implications for a Patagonia plate. Tectonics, 22: 5-33.

\section{NOTAS SOBRE LOS AUTORES}

Federico Lince Klinger. Licenciado en Geofísica en 2004 en la Universidad Nacional de San Juan, Argentina. Becario doctoral de la ANPCyT-F0NCyT en 20052008. Becario doctoral de CONICET desde 2008. Doctor en Geofísica en 2010 egresado de la Universidad Nacional de San Juan, Argentina. Profesor Titular de Geofísica Aplicada de la carrera Geología de la Universidad Nacional de La Rioja. Principales campos de investigación y docencia: Gravimetría y magnetometría.

Patricia Martinez. Licenciado en Geofísica en 1989, en la Universidad Nacional de San Juan. Doctor en Ingeniería en 1997, Universidad Nacional de Rosario. Investigador de CONICET desde 1997 al presente. Profesor de Geofísica del Instituto Geofísico Sismológico Volponi. Facultad de Ciencias Exactas, Físicas y Naturales de la Universidad Nacional de San Juan (UNSJ) desde 1993 hasta el presente. Principales campos de investigación y docencia: Gravimetría y magnetometría. 
Augusto Ernesto Rapalini. Licenciado en Ciencias Geológicas 1983, Universidad de Buenos Aires. Doctor en Ciencias Geológicas 1989, Universidad de Buenos Aires. Investigador Independiente de CONICET. Profesor Asociado Departamento de Ciencias Geológicas, FCEyN, UBA. Principales campos de investigación y docencia: Paleomagnetismo, Magnetofábrica, Paleogeografía, Geofísica Aplicada.

Mario Ernesto Gimenez. Licenciado en Geofísica en 1989, en la Universidad Nacional de San Juan. Doctor en Ingeniería en 1997, Universidad Nacional de Rosario. Investigador Adjunto de CONICET desde 2003 al presente. Profesor Titular en Geofísica General de la Carrera de Licenciatura en Geofísica desde 1999. Principales campos de investigación y docencia: Gravimetría y magnetometría.

Mónica Graciela López De Luchi. Licenciado en Ciencias Geológicas 1979, Universidad de Buenos Aires. Doctor en Ciencias Geológicas 1988, Universidad de Buenos Aires. Investigador Independiente de CONICET. Su tema de interés es la petrología de rocas ígneas y metamórficas en aspectos geoquímicos y microestructurales.

Flavia Anabel Croce. Licenciado en Geofísica en 1996, en la Universidad Nacional de San Juan. Doctor en Geofísica en 2006, Universidad Nacional de San Juan. Becaria de CONICET desde 2001 hasta 2007. Principales campos de investigación y docencia: Gravimetría y Geofísica aplicada a la criología.

Francisco Ruiz. Licenciado en Geofísica en 1993, en la Universidad Nacional de San Juan. Doctor en Ingeniería en 1998, Universidad Nacional de Rosario. Profesor adjunto en elementos de Geofísica de la Carrera de Ingeniería en Agrimensura de la Universidad Nacional de San Juan. Principales campos de investigación y docencia: Gravimetría, Geodesia y magnetometría. 